\title{
Urinary Creatinine Assay
}

National Cancer Institute

\section{Source}

National Cancer Institute. Urinary Creatinine Assay. NCI Thesaurus. Code C88225.

A laboratory test used for the measurement of creatinine levels in the urine to determine the degree of renal dysfunction. 